\title{
Fingerprint Verification Using Spectral Minutiae Representations
}

\author{
Haiyun Xu, Raymond N. J. Veldhuis, Asker M. Bazen, Tom A. M. Kevenaar, Ton A. H. M. Akkermans, and
}

Berk Gokberk

\begin{abstract}
Most fingerprint recognition systems are based on the use of a minutiae set, which is an unordered collection of minutiae locations and orientations suffering from various deformations such as translation, rotation, and scaling. The spectral minutiae representation introduced in this paper is a novel method to represent a minutiae set as a fixed-length feature vector, which is invariant to translation, and in which rotation and scaling become translations, so that they can be easily compensated for. These characteristics enable the combination of fingerprint recognition systems with template protection schemes that require a fixed-length feature vector. This paper introduces the concept of algorithms for two representation methods: the location-based spectral minutiae representation and the orientation-based spectral minutiae representation. Both algorithms are evaluated using two correlation-based spectral minutiae matching algorithms. We present the performance of our algorithms on three fingerprint databases. We also show how the performance can be improved by using a fusion scheme and singular points.
\end{abstract}

Index Terms-Biometrics, fingerprint recognition, minutiae matching, template protection.

\section{INTRODUCTION}

A MONG various biometric identifiers, such as face, signature, and voice, the fingerprint has one of the highest levels of distinctiveness and performance [1] and it is the most commonly used biometric modality. Compared with most other biometric techniques, fingerprint recognition systems also have the advantages of both ease of use and low cost. All these reasons explain the popularity of fingerprint recognition systems.

Minutiae are the endpoints and bifurcations of fingerprint ridges. They are known to remain unchanged over an individual's lifetime [1] and allow a very discriminative classification of fingerprints. Each minutia can be described by parameters $(x, y, \theta)$, where $(x, y)$ is the location of the minutia and $\theta$ its orientation [2].

Manuscript received December 18, 2007; revised March 08, 2009. First published April 28, 2009; current version published August 14, 2009. This work was supported by the research program Sentinels and conducted in cooperation with Philips Research Laboratories. The associate editor coordinating the review of this manuscript and approving it for publication was Dr. Tieniu Tan.

H. Xu, R. N. J. Veldhuis, and B. Gokberk are with the Department of Electrical Engineering, University of Twente, 7500 AE Enschede, The Netherlands (e-mail: h.xu@ewi.utwente.nl; r.n.j.veldhuis@ewi.utwente.nl; b.gokberk@ewi. utwente.nl).

A. M. Bazen is with Uniqkey Biometrics, 7546 KK Enschede, The Netherlands (e-mail: a.m.bazen@uniqkey.com).

T. A. M. Kevenaar is with priv-ID B.V., 5656 AE Eindhoven, The Netherlands (e-mail: tom.kevenaar@ priv-id.com).

T. A. H. M. Akkermans is with Philips Research Laboratories, 5656 AA Eindhoven, The Netherlands (e-mail: ton.h.akkermans@philips.nl).

Color versions of one or more of the figures in this paper are available online at http://ieeexplore.ieee.org.

Digital Object Identifier 10.1109/TIFS.2009.2021692
Today, many fingerprint recognition systems are based on minutiae matching [3], [4]. However, minutiae-based fingerprint matching algorithms have some drawbacks that limit their application. First, due to the fact that minutiae sets are unordered, the correspondence between individual minutia in two minutiae sets is unknown before matching and this makes it difficult to find the geometric transformation (consisting of translation, rotation, scaling, and optionally nonlinear deformations [4]) that optimally registers (or aligns) two sets. For fingerprint identification systems with very large databases [5], in which a fast comparison algorithm is necessary, minutiae-based matching algorithms will fail to meet the high performance speed requirements. Second, a minutiae representation of a fingerprint cannot be applied directly in recently developed template protection schemes based on fuzzy commitment and helper data schemes, such as [6] and [7], that require as an input a fixed-length feature vector representation of a biometric modality 1 .

The spectral minutiae representation as proposed in this paper overcomes the above drawbacks of the minutiae sets, thus broadening the application of minutiae-based algorithms. Our method is inspired by the Fourier-Mellin transform, which allows a representation of images in a way that is invariant to translation, rotation, and scaling [9]-[11]. By representing minutiae as a magnitude spectrum, we transform a minutiae set into a fixed-length feature vector that at the same time does not need registration to compensate for translation, rotation, and scaling. Our algorithm does not distinguish between endpoints and bifurcations, because the type of a minutia can be easily confused due to acquisition noises or pressure differences during acquisition. However, the orientation remains the same when this occurs. By using a spectral minutiae representation instead of minutiae sets, we meet the requirements of a template protection system and allow for faster matching as well.

The spectral minutiae representation method can be easily integrated into a minutiae-based fingerprint recognition system. Minutiae sets can be directly transformed to this new representation, which makes this method compatible with the large amount of existing minutiae databases.

This paper is organized as follows. First, the concept of spectral minutiae representation is explained in detail in Section II. Next, in Section III, two spectral minutiae matching algorithms are proposed. Then, Sections IV and V present the experimental results and discussions. Finally, we draw conclusions in Section VI.

\footnotetext{
${ }^{1}$ Other template protection systems exist [8] that do not pose this fixed-length feature vector requirement.
} 


\section{Spectral Minutiae Representation}

The spectral minutiae representation is based on the shift, scale, and rotation properties of the two-dimensional (2-D) continuous Fourier transform. If we have an input signal $f(\vec{x}), \vec{x}=$ $(x, y)^{T}$ (we denote the transpose of a vector $\vec{v}$ as $\vec{v}^{T}$ ), its continuous Fourier transform is

$$
F\{f(\vec{x})\}=F(\vec{\omega})=\int_{\vec{x} \in \mathbb{R}} f(\vec{x}) \exp \left(-j \vec{\omega}^{T} \vec{x}\right) d \vec{x}
$$

with $\vec{\omega}=\left(\omega_{x}, \omega_{y}\right)^{T}$. The Fourier transform of a translated $f(\vec{x})$ is

$$
F\left\{f\left(\vec{x}-\vec{x}_{0}\right)\right\}=\exp \left(-j \vec{\omega}^{T} \vec{x}_{0}\right) F(\vec{\omega})
$$

with $\vec{x}_{0}=\left(x_{0}, y_{0}\right)^{T}$ the translation vector. The Fourier transform of an isotropically scaled $f(\vec{x})$ is

$$
F\{f(a \vec{x})\}=a^{-2} F\left(a^{-1} \vec{\omega}\right)
$$

with $a(a>0)$ the isotropic scaling factor. The Fourier transform of a rotated $f(\vec{x})$ is

$$
F\{f(\Phi \vec{x})\}=F(\Phi \vec{\omega})
$$

with

$$
\Phi=\left(\begin{array}{cc}
\cos \phi & -\sin \phi \\
\sin \phi & \cos \phi
\end{array}\right)
$$

Here $\Phi$ is the (orthonormal) rotation matrix, and $\phi$ is the (counterclockwise) rotation angle of $f(\vec{x})$.

It can be seen from (2) that if only the magnitude of the Fourier spectrum is retained, this results in a translation invariant representation of the input signal. Furthermore, from (3) and (4), it follows that scaling and rotation of the input signal results in a scaled and rotated Fourier spectrum.

In order to exploit the above properties of the 2-D Fourier transform, we remap the Fourier spectral magnitude onto a polar-logarithmic coordinate system, such that the rotation and scaling become translations along the angular and radial axes, respectively. The detailed steps are as follows. Consider a signal $t(\vec{x})$ that is a translated, scaled, and rotated replica of $r(\vec{x})$

$$
t(\vec{x})=r\left(a \Phi \vec{x}-\vec{x}_{0}\right)
$$

then the magnitude of the Fourier transforms of $t(\vec{x})$ and $r(\vec{x})$ are related by

$$
|T(\vec{\omega})|=a^{-2}\left|R\left(a^{-1} \Phi \vec{\omega}\right)\right|
$$

which is a translation invariant representation of the input signal. If we remap the Fourier spectral magnitude onto a polar-logarithmic coordinate system as

$$
\begin{aligned}
\lambda & =\log \sqrt{\omega_{x}^{2}+\omega_{y}^{2}}, \quad \beta=\operatorname{angle}\left(\omega_{x}, \omega_{y}\right) \\
R_{\mathrm{pl}}(\lambda, \beta) & =\left|R\left(e^{\lambda} \cos \beta, e^{\lambda} \sin \beta\right)\right| \\
T_{\mathrm{pl}}(\lambda, \beta) & =\left|T\left(e^{\lambda} \cos \beta, e^{\lambda} \sin \beta\right)\right|
\end{aligned}
$$

then we have the Fourier spectral magnitude of $t(\vec{x})$ and $r(\vec{x})$ on the polar-logarithmic coordinates

$$
T_{\mathrm{pl}}(\lambda, \beta)=a^{-2} R_{\mathrm{pl}}(\beta+\phi, \lambda-\log a) .
$$

Equation (11) is a translation invariant description of the input signal, while the rotation and scaling have become translations along the new coordinate system axes. If we would perform a second Fourier transform on $T_{\mathrm{pl}}(\lambda, \beta)$, this is called a Fourier-Mellin transform [12], [13]. By retaining the magnitude of this Fourier-Mellin spectrum, we can obtain a translation, rotation, and scaling invariant representation of the input signal.

We will introduce a similar procedure as shown in (7)-(11), which can be applied to minutiae sets in order to find a representation that is invariant to translation and where rotation and scaling are translations.

\section{A. Location-Based Spectral Minutiae Representation (SML)}

When implementing the Fourier transform there are two important issues that should be considered. First, when a discrete Fourier transform is taken of an image, this results in a representation of a periodic repetition of the original image. This is undesirable because it introduces errors due to discontinuities at the image boundaries. Second, the remapping onto a polar-logarithmic coordinate system after using a discrete Fourier transform introduces interpolation artifacts. Therefore, we introduce an analytical representation of the input minutiae, and then use analytical expressions of a continuous Fourier transform that are evaluated on a grid in the polar-logarithmic plane. These analytical expressions are obtained as follows. Assume we have a fingerprint with $Z$ minutiae. With every minutia, a function $m_{i}(x, y)=\delta\left(x-x_{i}, y-y_{i}\right), i=1, \ldots, Z$ is associated where $\left(x_{i}, y_{i}\right)$ represents the location of the $i$ th minutia in the fingerprint image. Thus, in the spatial domain, every minutia is represented by a Dirac pulse. The Fourier transform of $m_{i}(x, y)$ is given by

$$
F\left\{m_{i}(x, y)\right\}=\exp \left(-j\left(\omega_{x} x_{i}+\omega_{y} y_{i}\right)\right)
$$

and the SML is defined as

$$
\mathcal{M}_{L}\left(\omega_{x}, \omega_{y}\right)=\sum_{i=1}^{Z} \exp \left(-j\left(\omega_{x} x_{i}+\omega_{y} y_{i}\right)\right) .
$$

In order to reduce the sensitivity to small variations in minutiae locations in the spatial domain, we use a Gaussian low-pass filter to attenuate the higher frequencies. This multiplication in the frequency domain corresponds to a convolution in the spatial domain where every minutia is now represented by a Gaussian pulse. A 2-D Gaussian $g(x, y)$ in the space domain and its Fourier transform $G\left(\omega_{x}, \omega_{y}\right)$ are

$$
\begin{aligned}
& g(x, y)=\frac{1}{2 \pi \sigma^{2}} \exp \left(-\frac{x^{2}+y^{2}}{2 \sigma^{2}}\right) \\
& \stackrel{F}{\longleftrightarrow} G\left(\omega_{x}, \omega_{y}\right)=\exp \left(-\frac{\omega_{x}^{2}+\omega_{y}^{2}}{2 \sigma^{-2}}\right) .
\end{aligned}
$$


Equation (14) shows that the parameter $\sigma$ of the Gaussian in the space domain appears as its reciprocal in the Gaussian in the frequency domain.

Following the shift property of the Fourier transform, the magnitude of $\mathcal{M}$ is taken in order to make the spectrum invariant to translation of the input and we obtain

$$
\begin{aligned}
& \left|\mathcal{M}_{L}\left(\omega_{x}, \omega_{y} ; \sigma_{L}^{2}\right)\right| \\
& \quad=\left|\exp \left(-\frac{\omega_{x}^{2}+\omega_{y}^{2}}{2 \sigma_{L}^{-2}}\right) \sum_{i=1}^{Z} \exp \left(-j\left(\omega_{x} x_{i}+\omega_{y} y_{i}\right)\right)\right| .
\end{aligned}
$$

Equation (15) is the analytical expression for the spectrum, which can directly be evaluated on a polar-logarithmic grid. The resulting representation in the polar-logarithmic domain is invariant to translation, while rotation and scaling of the input have become translations along the polar-logarithmic coordinates.

\section{B. Orientation-Based Spectral Minutiae Representation (SMO)}

The SML only uses the minutiae location information. However, including the minutiae orientation as well may give better discrimination. Therefore, it can be beneficial to also include the orientation information in our spectral representation. The orientation $\theta$ of a minutia can be incorporated by using the spatial derivative of $m(x, y)$ in the direction of the minutia orientation. Thus, to every minutia in a fingerprint, a function $m_{i}(x, y, \theta)$ is assigned being the derivative of $m_{i}(x, y)$ in the direction $\theta_{i}$, such that

$$
\begin{aligned}
F\left\{m_{i}(x, y, \theta)\right\}=j\left(\omega_{x} \cos \theta_{i}\right. & \left.+\omega_{y} \sin \theta_{i}\right) \\
\cdot & \exp \left(-j\left(\omega_{x} x_{i}+\omega_{y} y_{i}\right)\right) .
\end{aligned}
$$

As in the SML algorithm, using a Gaussian filter and taking the magnitude of the spectrum yields

$$
\begin{aligned}
& \left|\mathcal{M}_{O}\left(\omega_{x}, \omega_{y} ; \sigma_{O}^{2}\right)\right| \\
& =\mid \exp \left(-\frac{\omega_{x}^{2}+\omega_{y}^{2}}{2 \sigma_{O}^{-2}}\right) \sum_{i=1}^{Z} j\left(\omega_{x} \cos \theta_{i}+\omega_{y} \sin \theta_{i}\right) \\
& \quad \cdot \exp \left(-j\left(\omega_{x} x_{i}+\omega_{y} y_{i}\right)\right) \mid .
\end{aligned}
$$

\section{Implementation}

In the previous sections, we introduced analytical expressions for the spectral minutiae representations of a fingerprint. In order to obtain our final spectral representations, the continuous spectra (15) and (17) are sampled on a polar-logarithmic grid. In the radial direction $\lambda$, we use $M=128$ samples between $\lambda_{l}=0.1$ and $\lambda_{h}=0.6$. In the angular direction $\beta$, we use $N=256$ samples uniformly distributed between $\beta=0$ and $\beta=\pi$. Because of the symmetry of the Fourier transform for real-valued functions, using the interval between 0 and $\pi$ is sufficient. This polar-logarithmic sampling process is illustrated in Figs. 1 and 2.

The sampled spectra (15) and (17) will be denoted by $S_{L}\left(m, n ; \sigma_{L}\right)$ and $S_{O}\left(m, n ; \sigma_{O}\right)$, respectively, with

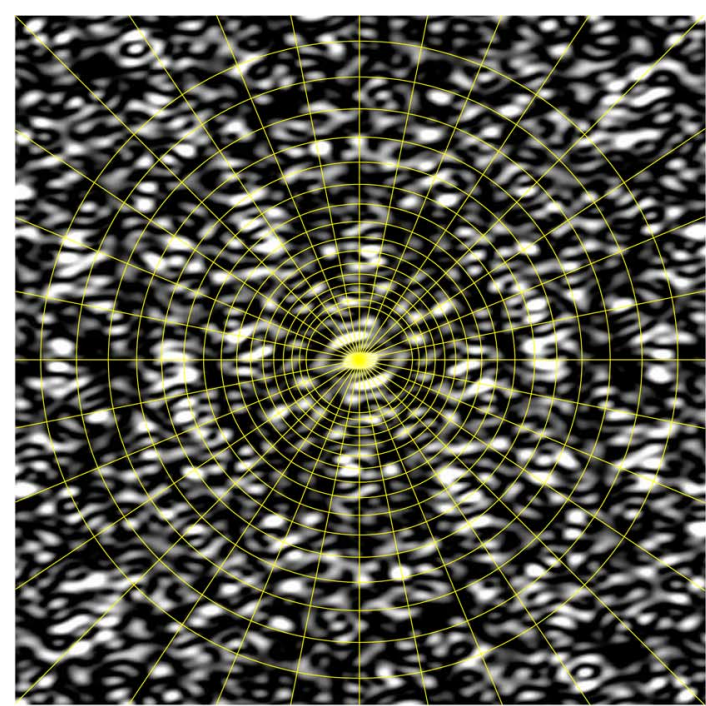

(a)

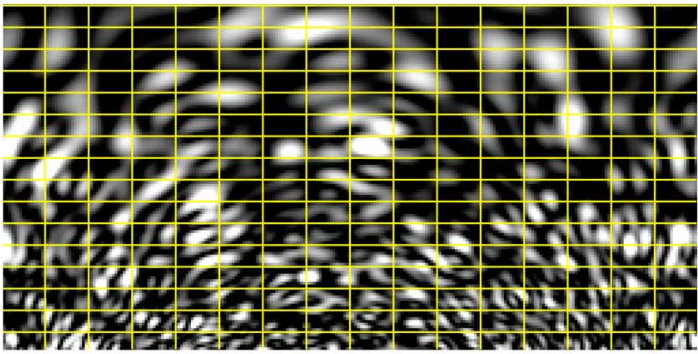

(b)

Fig. 1. Illustration of the polar-logarithmic sampling (SML spectra) (a) Fourier spectrum in a Cartesian coordinate and a polar-logarithmic sampling grid; (b) Fourier spectrum sampled on a polar-logarithmic grid.

$m=1, \ldots, M, n=1, \ldots, N$. When no confusion can arise, the parameter $\sigma$ and the subscripts $L$ and $O$ will be omitted.

Examples of the minutiae spectra achieved with SML are shown in Fig. 3, and those achieved with SMO are shown in Fig. 4. In these figures, $\sigma_{L}=0.32(15)$ and $\sigma_{O}=3.87$ (17). For each spectrum, the horizontal axis represents the rotation angle of the spectral magnitude (from 0 to $\pi$ ); the vertical axis represents the frequency of the spectral magnitude (the frequency increases from top to bottom). It should be noted that the minutiae spectrum is periodic on the horizontal axis.

\section{Spectral Minutiae Matching}

After representing fingerprints by minutiae spectra, the next step is matching: the comparison of two minutiae spectra. The result of matching is either a "match" (the two spectra appear to be from the same finger) or a "nonmatch" (the two spectra appear to be from different fingers). Normally, in this step, we will first compute a number (similarity score), which corresponds to the degree of similarity. Then, by using a threshold, we can make a match/nonmatch decision [14].

In this paper, two matching algorithms are presented. In the first algorithm (direct matching), the correlation of two spectral images was chosen as a similarity score, which is a common similarity measure in image processing. The second algorithm is the Fourier-Mellin matching, in which the Fourier transform 


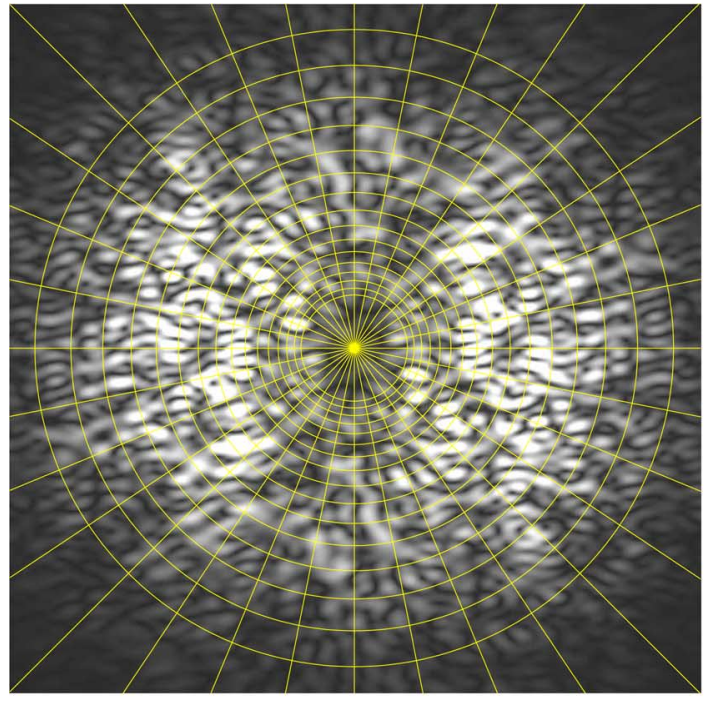

(a)

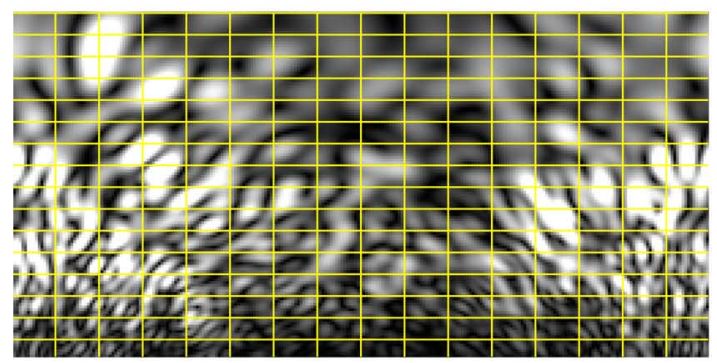

(b)

Fig. 2. Illustration of the polar-logarithmic sampling (SMO spectra). (a) Fourier spectrum in a Cartesian coordinate and a polar-logarithmic sampling grid; (b) Fourier spectrum sampled on a polar-logarithmic grid.

of the minutiae spectrum is taken, and only the magnitude is retained. This will generate a complete translation, rotation, and scaling invariant descriptor of the minutiae set, and then a correlation-based method is used to calculate the similarity score of the Fourier-Mellin spectra.

\section{A. Direct Matching}

Let $R(m, n)$ and $T(m, n)$ be the two sampled minutiae spectra in the polar-logarithmic domain, respectively, achieved from the reference fingerprint and test fingerprint. Both $R(m, n)$ and $T(m, n)$ are normalized to have zero mean and unit energy. We use the 2-D correlation coefficient between $R$ and $T$ as a measure of their similarity.

In practice, the input fingerprint images are rotated and might be scaled (for example, depending on the sensor that is used to acquire an image). Since the minutiae spectra are translation invariant but not rotation and scaling invariant, this method has to test a few different combinations of rotation and scaling, which are translations in the minutiae spectra. To be specific, the scaling becomes the shift (or translation) in the vertical direction, and the rotation becomes the circular shift in the horizontal direction. We denote $T(m-i, n-j)$ as a shifted version of $T(m, n)$, with a shift of $i$ in the vertical direction and a circular shift $j$ in the horizontal direction. Then, the correlation

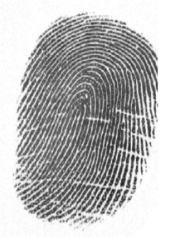

(a)

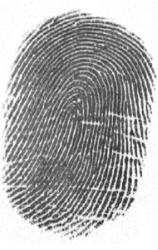

(c)

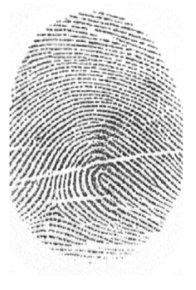

(e)

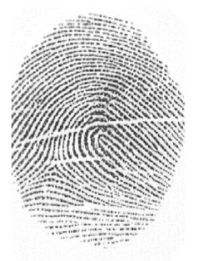

(g)

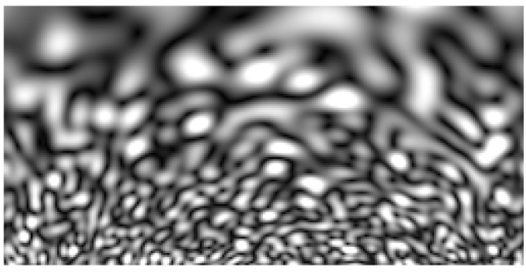

(b)

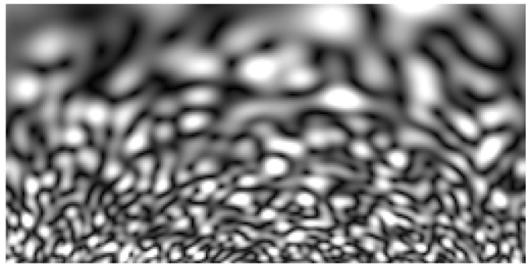

(d)

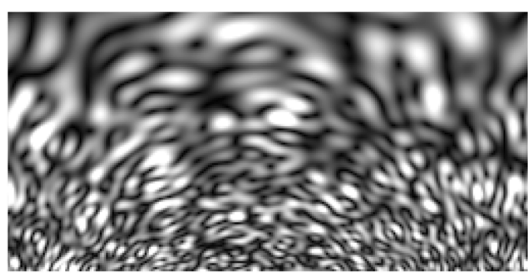

(f)

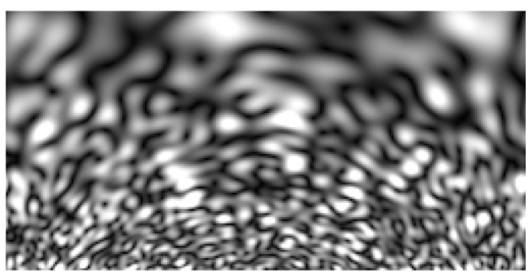

(h)
Fig. 3. Examples of minutiae spectra using SML. (a) and (c) are fingerprints from the same finger; (e) and (g) are fingerprints from the same finger. (b) Minutiae spectrum of (a). (d) Minutiae spectrum of (c). (f) Minutiae spectrum of (e). (h) Minutiae spectrum of $(\mathrm{g})$.

coefficient between $R$ and $T$ is defined as

$$
C^{(R, T)}(i, j)=\frac{1}{M N} \sum_{m, n} R(m, n) T(m-i, n-j)
$$

In most fingerprint databases, there is no scaling difference between the fingerprints, or the scaling can be compensated for on the level of the minutiae sets [15]. Therefore, in practice only a few rotations need to be tested. We chose to test rotations from -15 units to +15 units in steps of three units, which corresponds to a range from $-10^{\circ}$ to $+10^{\circ}$ in steps of $2^{\circ}$. The maximum score from the different combinations is the final matching score between $R$ and $T$

$$
S^{(R, T)}=\max _{j}\left\{C^{(R, T)}(0, j)\right\}
$$

with

$$
j=3 k, \quad \text { for } k=-5, \ldots, 5 \text {. }
$$




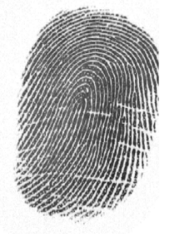

(a)

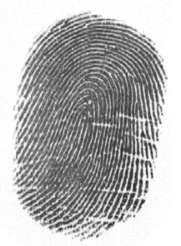

(c)

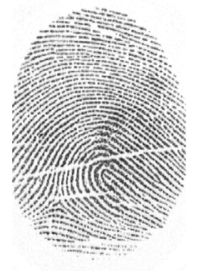

(e)

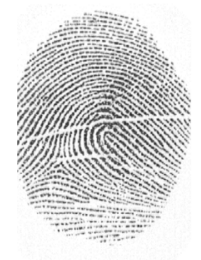

(g)

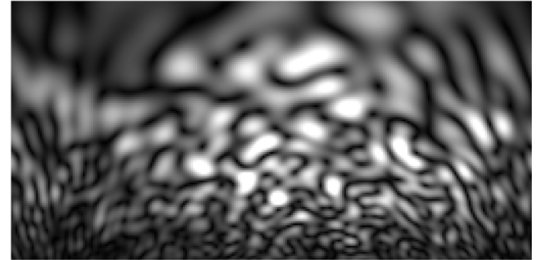

(b)

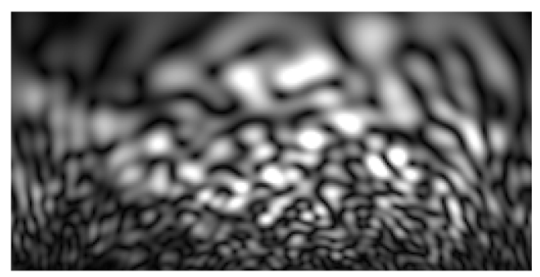

(d)

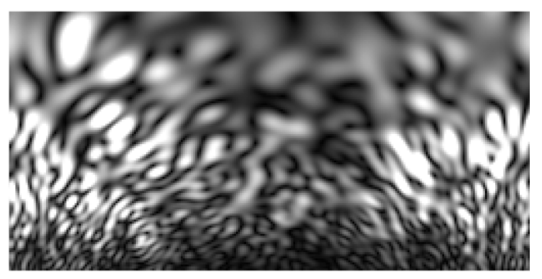

(f)

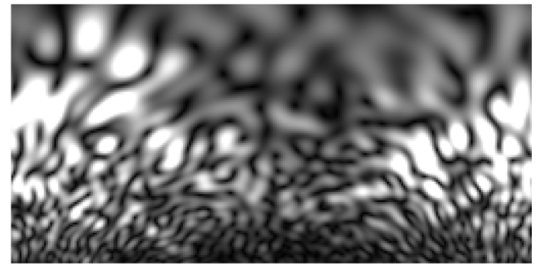

(h)
Fig. 4. Examples of minutiae spectra using SMO. (a) and (c) are fingerprints from the same finger; (e) and (g) are fingerprints from the same finger. (b) Minutiae spectrum of (a). (d) Minutiae spectrum of (c). (f) Minutiae spectrum of (e). (h) Minutiae spectrum of $(\mathrm{g})$.

\section{B. Fourier-Mellin Matching}

The Fourier-Mellin transform is often used to obtain a complete translation, rotation, and scaling invariant descriptor. It is based on the scale-invariance property of the Mellin transform. The Mellin transform [16] is defined for complex $s=\sigma+j \omega$ as

$$
M\{f(x)\}=F_{M}(s)=\int_{0}^{\infty} f(x) x^{s-1} d x .
$$

If we define the Mellin transform on the imaginary axis, thus $s=j \omega$, then the Mellin transform becomes

$$
F_{M}(\omega)=\int_{0}^{\infty} f(x) x^{j \omega-1} d x
$$

The Mellin transform of a scaled $f(x)$ with a scaling factor $a$ is

$$
M\{f(a x)\}=F_{M}^{a}(\omega)=\int_{0}^{\infty} f(a x) x^{j \omega-1} d x .
$$

If we make a change of variable $y=a x$, thus $x=y / a$, then (23) becomes

$$
\begin{aligned}
F_{M}^{a}(\omega) & =\int_{0}^{\infty} f(y)\left(\frac{y}{a}\right)^{j \omega-1} \frac{1}{a} d y \\
& =a^{-j \omega} \int_{0}^{\infty} f(y) y^{j \omega-1} d y \\
& =a^{-j \omega} F_{M}(\omega) \\
& =\exp (-j \omega \ln a) F_{M}(\omega) .
\end{aligned}
$$

Equation (24) shows that the scale change in the time domain just becomes a phase change in the Mellin domain. Therefore, the magnitude of the Mellin transform is scale-invariant

$$
\left|F_{M}^{a}(\omega)\right|=\left|F_{M}(\omega)\right|
$$

A standard Fourier-Mellin transform, sometimes called a circular Fourier and radial Mellin transform [17], is written as

$$
\begin{aligned}
M_{f}\{f(r, \beta)\} & =M_{f}\left(s, \omega_{\beta}\right) \\
& =\int_{0}^{2 \pi} \int_{0}^{\infty} r^{s-1} f(r, \beta) \exp \left(-j \omega_{\beta} \beta\right) d r d \beta .
\end{aligned}
$$

If we make a change of variable $r=e^{\lambda}$, thus $\lambda=\ln r$, and let $s=-j \omega_{\lambda}$, thus defining the radial Mellin transform on the imaginary axis, then the Fourier-Mellin transform becomes

$$
\begin{aligned}
M_{f} & \{f(r, \beta)\} \\
& =M_{f}\left(\omega_{\lambda}, \omega_{\beta}\right) \\
& =\int_{0}^{2 \pi} \int_{-\infty}^{\infty} f(\lambda, \beta) \exp \left(-j \omega_{\lambda} \lambda\right) \exp \left(-j \omega_{\beta} \beta\right) d \lambda d \beta .
\end{aligned}
$$

This is a 2-D Fourier transform of the function $f(\lambda, \theta)$. Equation (27) shows that the Fourier-Mellin transform can be implemented by a polar-logarithmic transform of the original signal, and then using a 2-D Fourier transform. Therefore, by performing a 2-D Fourier transform on the minutiae spectra, we implement a Fourier-Mellin transform, and we can obtain a Fourier-Mellin descriptor by only retaining the magnitude. We denote $R_{\mathrm{FM}}(m, n)$ and $T_{\mathrm{FM}}(m, n)$ as the magnitude of the 2-D Fourier transform of the spectral minutiae spectra $R(m, n)$ and $T(m, n)$. In the Fourier-Mellin matching algorithm, the correlation of two Fourier-Mellin magnitude $R_{\mathrm{FM}}(m, n)$ and $T_{\mathrm{FM}}(m, n)$ was chosen as a similarity score

$$
S_{\mathrm{FM}}^{(R, T)}=\frac{1}{M N} \sum_{m, n} R_{\mathrm{FM}}(m, n) T_{\mathrm{FM}}(m, n) .
$$

\section{EXPERIMENTS}

\section{A. Measurements}

We test the spectral minutiae representation in a verification setting. A verification system authenticates a person's identity by comparing the captured biometric characteristic with the corresponding biometric template(s) prestored in the system. 
TABLE I

CHARACTERISTICS OF DATABASES USED IN OUR EXPERIMENTS

\begin{tabular}{|c|c|c|c|}
\hline & MCYT & FVC2000-DB2 & FVC2002-DB2 \\
\hline Sensor & $\begin{array}{c}\text { U.are.U } \\
\text { (Digital Persona) }\end{array}$ & $\begin{array}{c}\text { TouchChip } \\
\text { (ST Microelectronics) }\end{array}$ & $\begin{array}{c}\text { FX2000 } \\
\text { (Biometrika) }\end{array}$ \\
\hline Sensor type & optical & capacitive & optical \\
\hline Image size & $256 \times 400$ & $256 \times 364$ & $296 \times 560$ \\
\hline Resolution & $500 \mathrm{dpi}$ & $500 \mathrm{dpi}$ & $569 \mathrm{dpi}$ \\
\hline
\end{tabular}

It conducts a one-to-one comparison to determine whether the identity claimed by the individual is true [1].

The matching performance of a fingerprint verification system can be evaluated by means of several measures. Commonly used are the false acceptance rate (FAR), the false rejection rate (FRR), and the equal error rate (EER). When the decision threshold of a biometric security system is set such that the FAR and FRR are equal, the common value of FAR and FRR is referred to as the EER. In this paper, we use FAR, $\mathrm{EER}$, and the genuine accept rate (GAR), GAR $=1-\mathrm{FRR}$, as performance indicators of our scheme.

\section{B. Experimental Settings}

The proposed algorithms have been evaluated on MCYT [18], FVC2000-DB2 [19], and FVC2002-DB2 [20] fingerprint databases. The fingerprint data that we used from MCYT are obtained from ten individuals. Each individual contributed data from ten different fingers with ten samples per finger. We also tested our algorithms on two FVC fingerprint databases because they are public-domain fingerprint databases. Compared with MCYT, the fingerprints in FVC have lower quality and bigger displacements. For the FVC databases, we used the same experimental protocol as in the FVC competition. Both FVC databases contain 100 fingers, with eight samples per finger. In FVC2002-DB2, we only used four samples (samples 1, 2, 7, and 8) in our experiments ${ }^{2}$, while in FVC2000-DB2, we used all eight samples from each finger. The characteristics of the databases are summarized in Table I.

We generated two minutiae sets from MCYT. The first minutiae set contains manually extracted minutiae, which serves as a high-quality minutiae set. The second minutiae set is obtained by the VeriFinger minutiae extractor [21] and will be called "VeriFinger minutiae." In order to be able to manually extract reliable minutiae from fingerprint samples, we chose the ten individuals from MCYT that have reasonably good quality fingerprints. The quality measurement that we used here is based on the fingerprint's variance and coherence [22]. The variance and the coherence of a fingerprint reflect the clarity of its ridge-valley structures. In general, good-quality fingerprints have higher variance and coherence than low-quality fingerprints. Some samples that we accepted and rejected from MCYT are shown in Fig. 5. For FVC databases, we only used the minutiae sets that are obtained by the VeriFinger minutiae extractor.

${ }^{2}$ In FVC2002 databases, samples 3, 4, 5, and 6 were obtained by requesting the users to provide fingerprints with exaggerated displacement and rotation [8]. In a security scenario where the user is aware that cooperation is crucial for security reasons, he will be cooperative. Therefore, only samples $1,2,7$, and 8 are chosen.

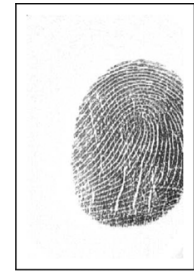

(a)

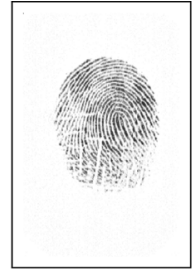

(b)

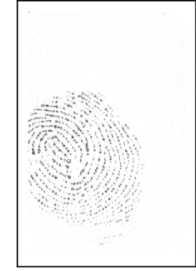

(c)

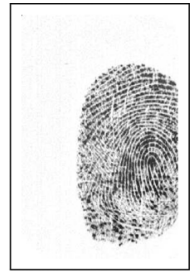

(d)
Fig. 5. Examples of fingerprint samples in MCYT: (a) and (b) are the fingerprints that we accepted from MCYT; (c) and (d) are fingerprints that we rejected from MCYT because of the low quality.

For each comparison, we chose two fingerprints from the data set: one as a reference fingerprint, another as a test fingerprint. For matching genuine pairs, we used all the possible combinations. For matching imposter pairs, we chose one sample from each identity. Therefore, we have 4500, 2800, and 600 genuine scores for MCYT, FVC2000-DB2, and FVC2002-DB2, respectively. For each database, we have 4950 imposter scores.

In the spectral minutiae representation, we used a Gaussian low-pass filter on the spectrum to attenuate the higher frequencies [see (15) and (17)]. From our experiments, we noticed that for SML and SMO, we need to choose different Gaussian parameters $\left(\sigma_{L}\right.$ and $\left.\sigma_{O}\right)$ to achieve the best performances. Figs. 6 and 7 show the influence of the Gaussian parameter $\sigma$ to the performances on MCYT VeriFinger minutiae set (using direct matching algorithm). We noticed that the Gaussian parameter has larger effects on SML than on SMO. Moreover, a Gaussian kernel is needed for SMO for achieving a better performance, while for SML it is not. The reason is that because the minutiae orientation is incorporated as a derivative of the delta function [see (16)], this will amplify the noise (both in minutiae location and orientation) in the high frequency part in SMO. Therefore, a Gaussian kernel is needed for SMO to attenuate the higher frequencies. In SML, the high frequency part contains discriminative information, while the noise is evenly distributed in all frequencies; therefore, a Gaussian kernel does not help for a better performance. In our experiments, we finally chose $\sigma=0$ for SML (in this case, no multiplication with Gaussian in the frequency domain) and $\sigma=4.24$ for SMO. In case the fingerprint resolution is $500 \mathrm{dpi}$, the Gaussian parameter $\sigma=4.24$ (pixel) in the spacial domain is about $0.21(\mathrm{~mm})$ in reality.

From our experiments, we also noticed that the careful selection of frequency ranges $\left(\lambda_{l}\right.$ and $\left.\lambda_{h}\right)$ of spectral minutiae are essential for a high performance, especially for SMO. For low quality fingerprints or an unreliable minutiae extractor (where the errors on minutiae location and orientation are higher), we need to use the lower frequencies that are more robust to noise. The final settings of $\lambda_{l}$ and $\lambda_{h}$ for the databases are shown in Table II.

\section{Results of SML and SMO}

We tested both SML and SMO representation methods. The EERs we achieved are shown in Tables III $-\mathrm{V}$, and the receiver operating characteristic (ROC) curves are shown in Figs. 8-10. For MCYT VeriFinger minutiae sets, the genuine and imposter distributions (resulting from direct matching) are shown in Fig. 11. 


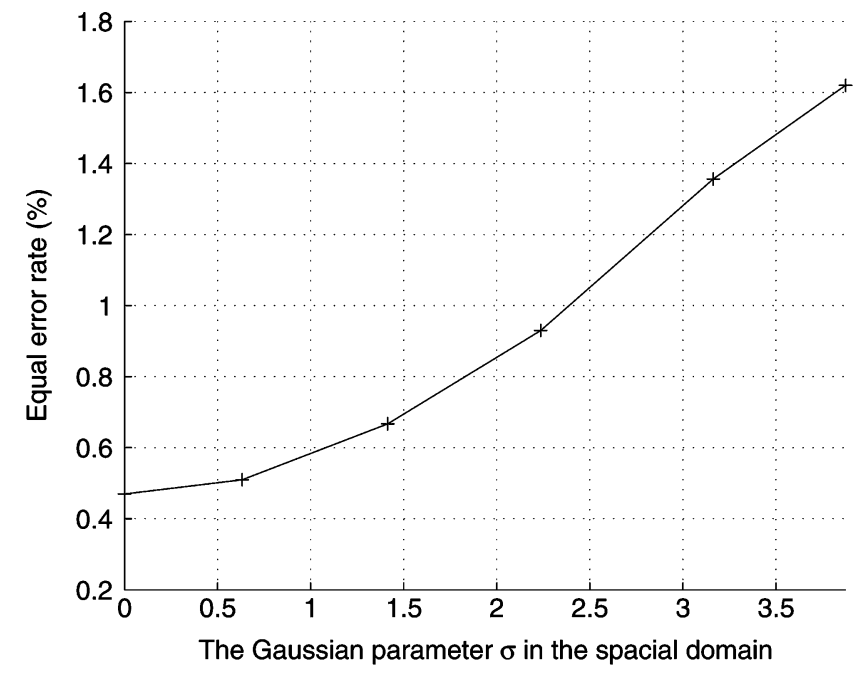

Fig. 6. Relation of the Gaussian $\sigma$ (in the spacial domain) and SML performances (MCYT VeriFinger minutiae set, using direct matching algorithm).

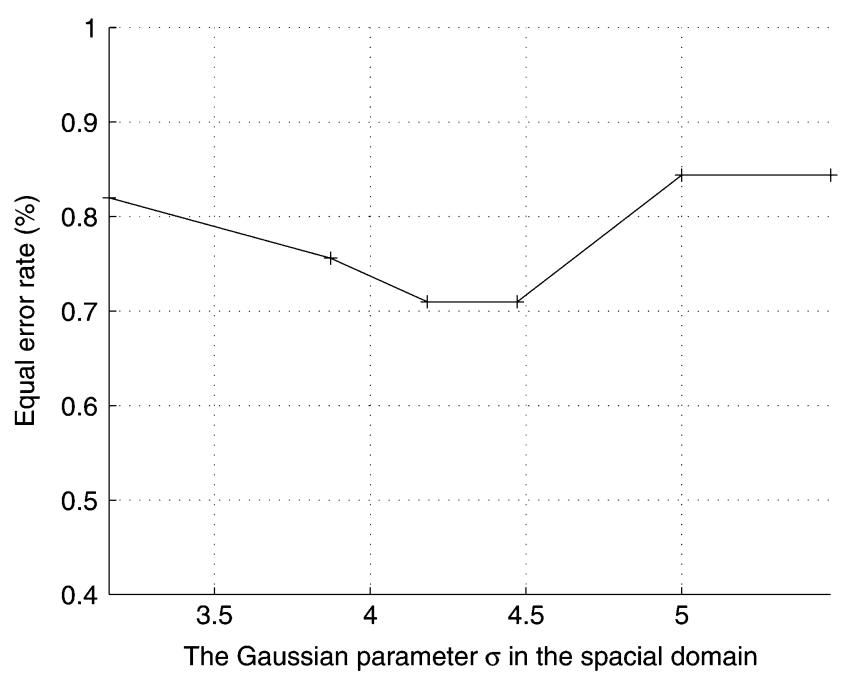

Fig. 7. Relation of the Gaussian $\sigma$ (in the spacial domain) and SMO performances (MCYT VeriFinger minutiae set, using direct matching algorithm).

From Tables III-V, we can see that the direct matching algorithm received better results than the Fourier-Mellin matching algorithm. The Fourier-Mellin matching algorithm first implemented a 2-D Fourier transform, and then achieved a rotation and scaling invariant descriptor by only retaining the magnitude. In this step, the phase information was discarded. However, in our application, the spectral minutiae do not suffer from the scaling problem, and the rotation range is also limited. From the result, we can see that by discarding phase to achieve this rotation and scaling invariant degraded the performance. For the direct matching algorithm, SML received better results if the minutiae are with high quality (MCYT manual minutiae case). When using automatically extracted minutiae sets (in which the minutiae suffer more noise), SMO performed better.

From the results, we can also see that for both SMO and SML, the manually extracted minutiae received better results than the VeriFinger minutiae for MCYT. Also, MCYT received much better results than the two FVC databases. These show that our algorithms are sensitive to the minutiae quality and fingerprint
TABLE II

SETTINGS OF THE FREQUENCY RANGE

\begin{tabular}{|l|c|c|c|c|}
\hline \multirow{2}{*}{ Databases } & \multicolumn{2}{|c|}{ SML } & \multicolumn{2}{c|}{ SMO } \\
\cline { 2 - 5 } & $\lambda_{\mathrm{l}}$ & $\lambda_{\mathrm{h}}$ & $\lambda_{\mathrm{l}}$ & $\lambda_{\mathrm{h}}$ \\
\hline MCYT (Manual minutiae) & 0.1 & 0.6 & 0.05 & 0.6 \\
\hline MCYT (VeriFinger minutiae) & 0.1 & 0.6 & 0.01 & 0.56 \\
\hline FVC2000-DB2 and FVC2002-DB2 & 0.08 & 0.62 & 0.001 & 0.53 \\
\hline
\end{tabular}

TABLE III

MCYT: DIRECT MATCHING RESULTS

\begin{tabular}{|l|c|c|}
\hline Minutiae sets & EERs (SML) & EERs (SMO) \\
\hline Manual minutiae & $0.09 \%$ & $0.12 \%$ \\
\hline VeriFinger minutiae & $0.47 \%$ & $0.42 \%$ \\
\hline
\end{tabular}

TABLE IV

MCYT: FOURIER-MELLIN MATCHING RESULTS

\begin{tabular}{|l|c|c|}
\hline Minutiae sets & EERs (SML) & EERs (SMO) \\
\hline Manual minutiae & $3.16 \%$ & $1.96 \%$ \\
\hline VeriFinger minutiae & $6.56 \%$ & $3.29 \%$ \\
\hline
\end{tabular}

TABLE V

FVC: DIRECT MATCHING RESUlTS

\begin{tabular}{|l|c|c|}
\hline Databases & EERs (SML) & EERs (SMO) \\
\hline FVC2000-DB2 & $9.8 \%$ & $8.4 \%$ \\
\hline FVC2002-DB2 & $6.4 \%$ & $6.1 \%$ \\
\hline
\end{tabular}

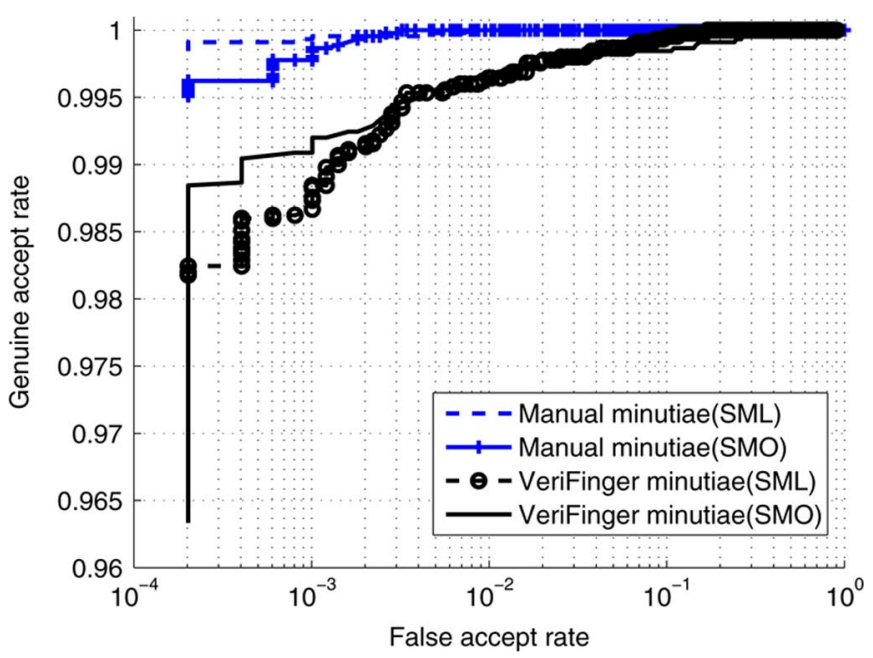

Fig. 8. ROC curves (MCYT: using direct matching).

quality. In Section V, we will present a further discussion about the factors that can influence the performance of our algorithms.

\section{Fusion Results of SML and SMO}

In Section IV-C, we showed the recognition results for both SML and SMO. To illustrate the relation of the SML and SMO results, we made a scatter plot for the genuine and imposter scores in the MCYT manual minutiae case (direct matching results), shown in Fig. 12.

From Fig. 12, we can see that the genuine scores from SML and SMO are correlated. At the same time, the imposter scores seem relatively uncorrelated. From the picture it can also be seen 


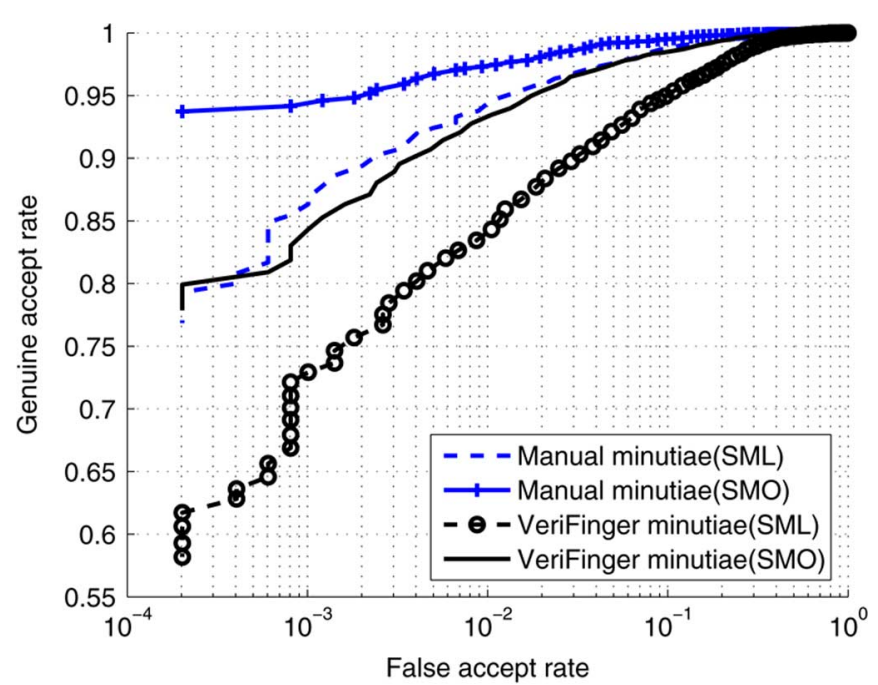

Fig. 9. ROC curves (MCYT: using Fourier-Mellin matching).

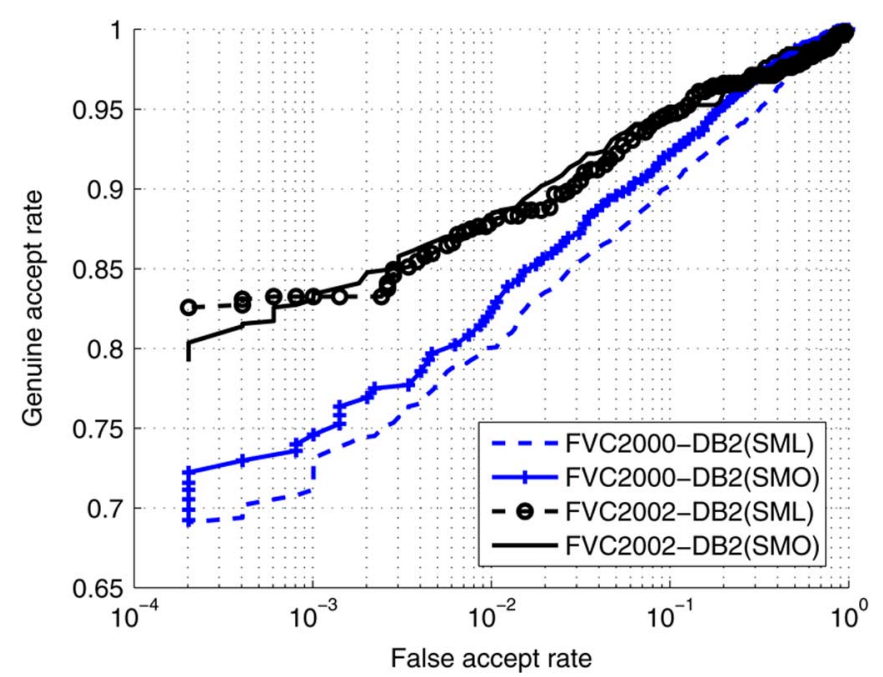

Fig. 10. ROC curves (FVC2000-DB2 and FVC2002-DB2: using direct matching).

that the genuine and imposter distribution can be better separated using a fusion approach [23]. For this reason, we use the matching score based fusion to improve the performance. A sum rule with equal weights for SML and SMO is used [24], whose decision boundary is shown as the dashed line in Fig. 12. The fusion results are shown in Tables VI-VIII (for comparison, the results of SML and SMO are also listed). The ROC curves are shown in Figs. 13 and 14 (using the direct matching algorithm). We can see that the fusion results are much better than the SML and SMO results.

For fingerprint identification systems with very large databases, the matching speed is crucial. Because our algorithms use a fixed-length feature vector and avoid fingerprint alignment, the matching speed is promising. For both SML and SMO using the direction matching algorithm, we need to implement 360448 real multiplications and 360437 real additions. We tested the matching speed for the fusion case of SML and SMO and we can implement 8000 comparisons (or matchings)

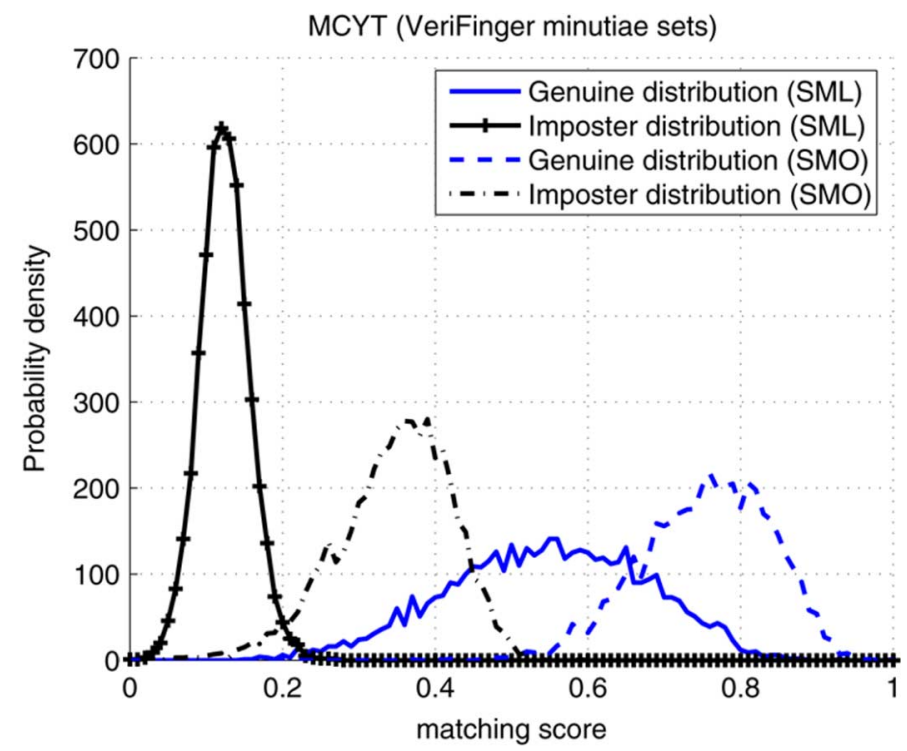

Fig. 11. Genuine and imposter distributions (VeriFinger minutiae sets, using direct matching).

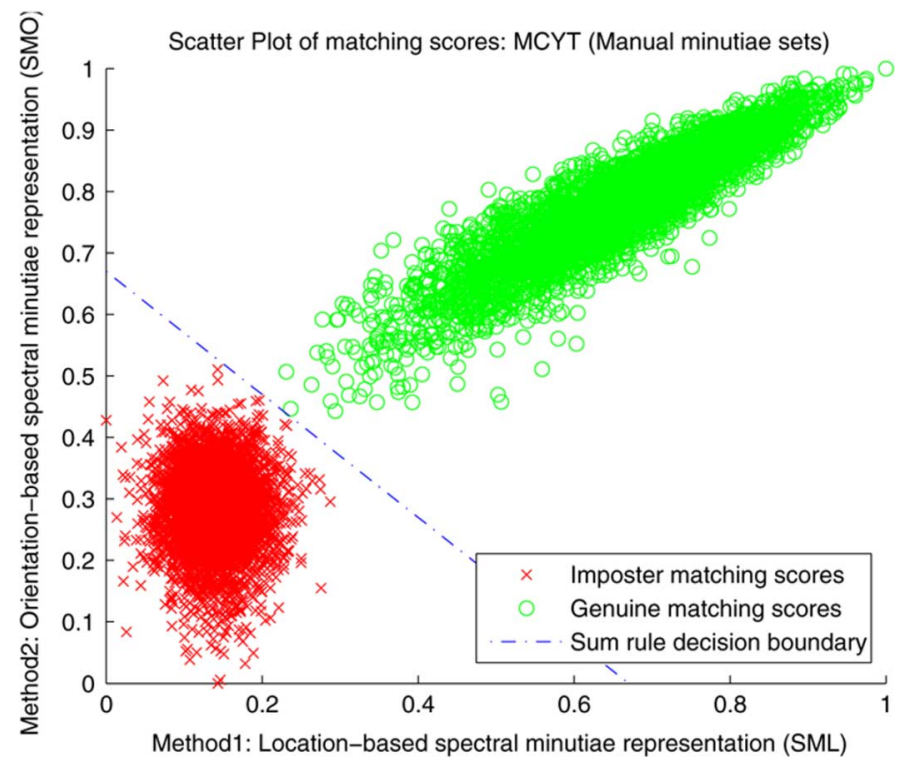

Fig. 12. Scatter plot of scores in MCYT manual minutiae case.

TABLE VI

MCYT: FUSION RESULTS OF SML AND SMO (DIRECT MATCHING)

\begin{tabular}{|l|c|c|c|}
\hline Minutiae sets & EERs (SML) & EERs (SMO) & EERs (fusion) \\
\hline Manual minutiae & $0.09 \%$ & $0.12 \%$ & $0 \%$ \\
\hline VeriFinger minutiae & $0.47 \%$ & $0.42 \%$ & $0.22 \%$ \\
\hline
\end{tabular}

TABLE VII

MCYT: FUSION RESULTS OF SML AND SMO (FOURIER-MELLIN MATCHING)

\begin{tabular}{|l|c|c|c|}
\hline Minutiae sets & EERs (SML) & EERs (SMO) & EERs (fusion) \\
\hline Manual minutiae & $3.16 \%$ & $1.96 \%$ & $1.0 \%$ \\
\hline VeriFinger minutiae & $6.56 \%$ & $3.29 \%$ & $1.86 \%$ \\
\hline
\end{tabular}

per second using optimized $\mathrm{C}$ language programming on a $\mathrm{PC}$ with Intel Pentium D processor $2.80 \mathrm{GHz}$ and $1 \mathrm{~GB}$ of RAM. 
TABLE VIII

FVC: FUSION RESULTS OF SML AND SMO (DIRECT MATCHING)

\begin{tabular}{|l|c|c|c|}
\hline Databases & EERs (SML) & EERs (SMO) & EERs (fusion) \\
\hline FVC2000-DB2 & $9.8 \%$ & $8.4 \%$ & $6.2 \%$ \\
\hline FVC2002-DB2 & $6.4 \%$ & $6.1 \%$ & $4.8 \%$ \\
\hline
\end{tabular}

\section{E. Improvements by Using Singular Points of Fingerprints}

In a fingerprint, singular points (SPs) can be identified. Henry [25] defined two types of SPs, the core and the delta. One fingerprint contains $0-2$ cores and $0-2$ deltas. Some methods used SPs to pre-align the fingerprints [6], [26]. However, some fingerprints do not contain SPs. Therefore, using SPs for pre-alignment is not reliable. In Table IX, we show the percentages of fingerprints in which SPs are not detected by the VeriFinger minutiae extractor. There are three cases for the failure of SPs detection: 1) the finger does not contain SPs; 2) SPs are not present in the fingerprint image because of a big displacement; and 3) the extractor fails to find the SPs. If we only accept the fingerprints containing SPs, it will cause a high failure-to-capture rate (FTCR). Therefore, we have designed an algorithm in which SPs can assist the verification, while they will not cause FTCR.

In our algorithm, SPs are used to avoid the limited-overlap problems between the reference and test fingerprints. We use the direct matching algorithm for the experiment and the matching score without SP information is denoted as $S_{\mathrm{DM}}$. Taking SML as an example, the procedure is as follows.

1) In a minutiae set, only take the minutiae within distance $D$ from the SPs as a new minutiae set to generate a subset spectral minutiae representation (sub-SM). Thus, depending on the number of SPs, each fingerprint can generate 0-4 different sub-SMs.

2) During matching, if both the reference and test fingerprints have the same type of SPs (both having at least 1 core or 1 delta), 1-4 comparisons (the matching scores are denoted as $S_{\mathrm{SP} 1-4}$ ) will be generated. For example, if the reference fingerprint has one core, while the test fingerprint has two, the sub-SM from the reference fingerprint will be compared with each of the sub-SMs from the test fingerprint. If the reference and test fingerprints have both two cores, two comparisons will be generated by comparing the sub-SMs from the upper-left core, and the sub-SMs from the lower-right core, respectively.

3) The largest score among $S_{\mathrm{DM}}$ and $S_{\mathrm{SP} 1-4}$ is chosen as the new matching score, denoted as $S_{\mathrm{SP}}$.

4) A sum-rule fusion as presented in Section IV-D for $S_{\mathrm{DM}}$ and $S_{\mathrm{SP}}$ is used as the final result for SML.

Finally, the steps 1-4 are also applied to SMO, and a sum-rule fusion as presented in Section IV-D for SML and SMO is used for a final result using SPs. We performed the experiments on the three databases (using VeriFinger minutiae sets). Considering the resolution and image size of fingerprints from each database (see Table I), we chose the distance $D=120$ (pixel) for MCYT and FVC2000-DB2, and $D=130$ (pixel) for FVC2002-DB2. The results are shown in Table $\mathrm{X}$ and the ROC curves of FVC databases are shown in Fig. 15 (for comparison, the ROC curves without SPs improvement are also shown).

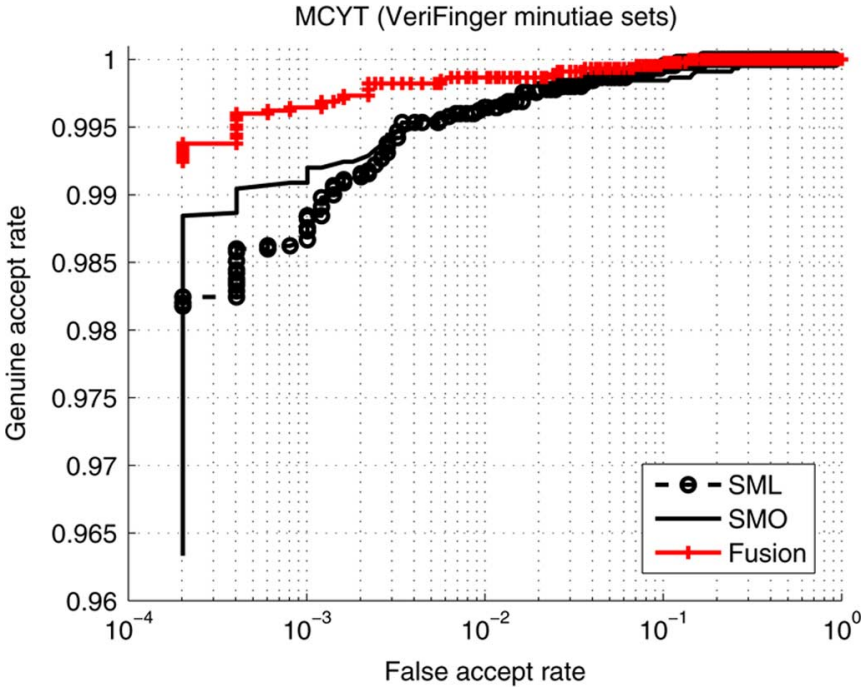

Fig. 13. ROC curves (MCYT: using direct matching).

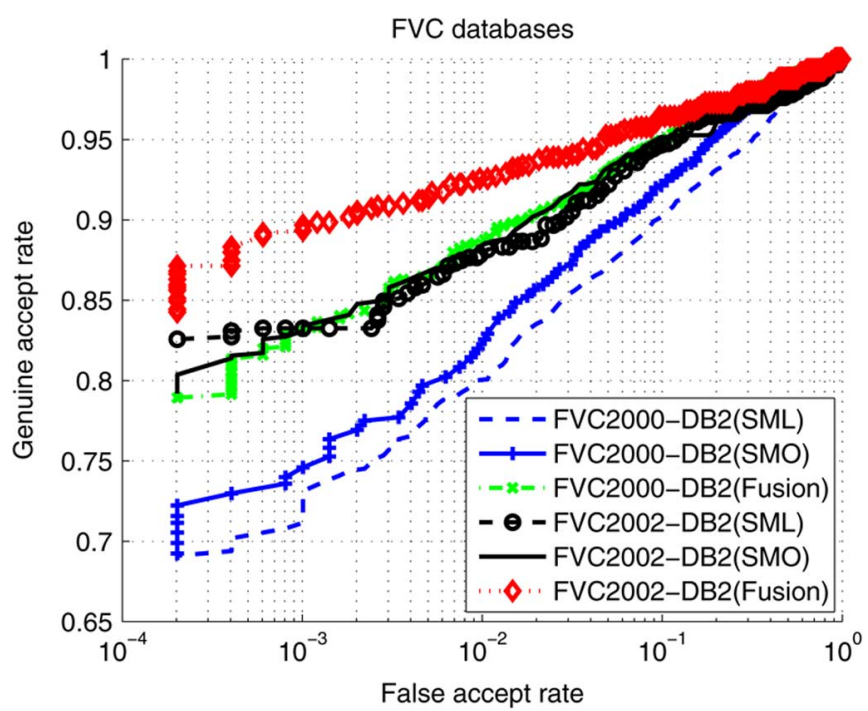

Fig. 14. ROC curves (FVC: using direct matching).

TABLE IX

Percentages of Fingerprints Without SPs

\begin{tabular}{|l|c|c|c|}
\hline Databases & No core & No delta & No core and no delta \\
\hline MCYT & $4.8 \%$ & $73 \%$ & $3.6 \%$ \\
\hline FVC2000-DB2 & $18 \%$ & $81.5 \%$ & $16.25 \%$ \\
\hline FVC2002-DB2 & $7.75 \%$ & $66 \%$ & $7.75 \%$ \\
\hline
\end{tabular}

TABLE $X$

REsults Using SPs (DiRECT MATChING)

\begin{tabular}{|l|c|c|c|c|}
\hline \multirow{2}{*}{ Databases } & \multirow{2}{*}{ EER } & \multicolumn{3}{|c|}{ GAR } \\
\cline { 3 - 5 } & & FAR $=1 \%$ & FAR $=0.1 \%$ & FAR $=0 \%$ \\
\hline MCYT & $0.13 \%$ & $99.9 \%$ & $99.8 \%$ & $99.5 \%$ \\
\hline FVC2000-DB2 & $5.19 \%$ & $91.7 \%$ & $88.1 \%$ & $85.5 \%$ \\
\hline FVC2002-DB2 & $3.86 \%$ & $95.5 \%$ & $92.7 \%$ & $89.7 \%$ \\
\hline
\end{tabular}




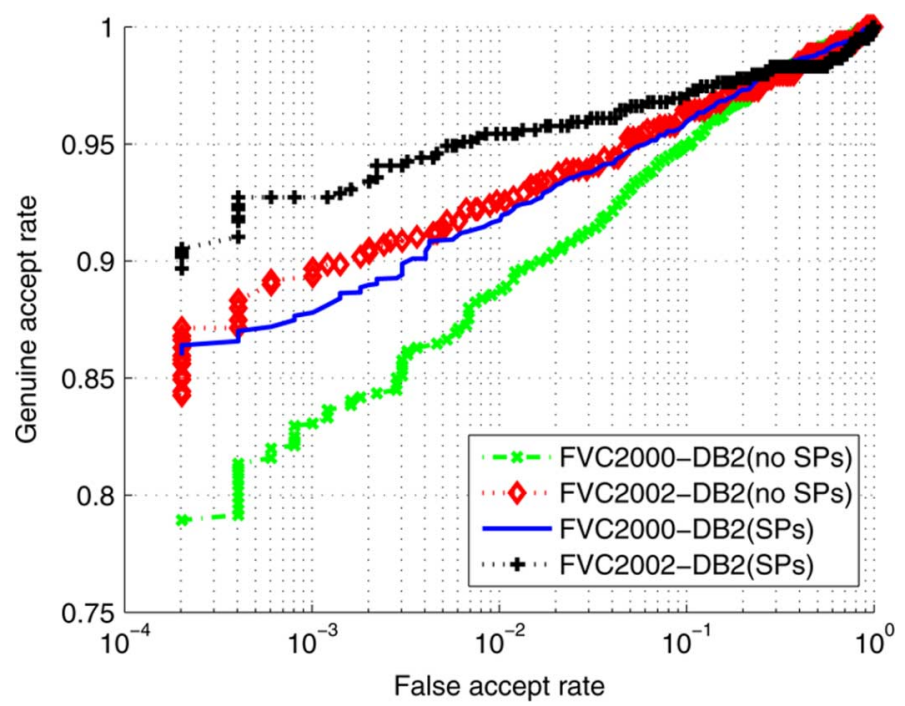

Fig. 15. ROC curves using SPs.

TABLE XI

RESULTS COMPARISON ON FVC2002-DB2

\begin{tabular}{|l|c|c|c|c|}
\hline \multirow{2}{*}{ Databases } & \multirow{2}{*}{ EER } & \multicolumn{3}{|c|}{ GAR } \\
\cline { 3 - 5 } & & FAR $=1 \%$ & FAR $=0.1 \%$ & FAR $=0 \%$ \\
\hline Our method & $3.86 \%$ & $95.5 \%$ & $92.7 \%$ & $89.7 \%$ \\
\hline VeriFinger & $1.0 \%$ & $99 \%$ & $98.8 \%$ & $98.6 \%$ \\
\hline Fuzzy Vault & - & - & $91 \%$ & $86 \%$ \\
\hline
\end{tabular}

\section{F. Comparison}

We compared our results with other well-known minutiae matchers on the FVC2002-DB2 database: VeriFinger ${ }^{3}$ and Fuzzy Vault according to the protocol in [8]. The results are shown in Table XI. We notice that the commercial minutiae matcher VeriFinger received much better results than ours. One reason is that the VeriFinger matcher uses some fingerprint features that are not defined in ISO minutiae template [15]. Another reason is that, with our global representation, we cannot perform minutiae pair searching, which is a crucial step for minutiae-based matching. These two reasons may cause the degradation of our algorithm compared with VeriFinger.

We also compared the performance of our method with a minutiae-based fingerprint recognition system combined with a template protection scheme based on fuzzy vault, which is presented in [8]. The reason of this comparison is that in [8] an alignment between a fingerprint pair using minutiae information is also not possible. Please note that [8] includes a template protection scheme, whereas our system does not. Moreover, because [8] implemented an alignment using high curvature points derived from the fingerprint orientation field, this caused a $2 \%$ FTCR, while our method does not suffer from this.

\section{DISCUSSION}

The spectral minutiae representations are minutiae-based algorithms. A false rejection from a minutiae-based fingerprint recognition system can be caused by several factors: 1) translation, rotation, and scaling deformations of minutiae; 2) non-

\footnotetext{
${ }^{3}$ VeriFinger Extractor Version 5.0.2.0 and VeriFinger Matcher version 5.0.2.1 are used.
}

linear distortions of minutiae; 3 ) limited overlap between the reference and test fingerprints; 4) missing minutiae (the minutiae extractor fails to detect the existing minutiae) and spurious minutiae (the minutiae extractor falsely identifies a minutia); and 5) errors on minutiae location and orientation.

The spectral minutiae matching algorithms have been designed to cope with the minutiae translation, rotation and scaling deformations (factor 1). Table VI shows that the manually extracted minutiae receive a better recognition accuracy than the automatically extracted minutiae (VeriFinger minutiae sets). We assume that the manual extraction is accurate to detect the true minutia and obtain its location and orientation, then the performance degradation of the automatically extracted minutiae is mainly caused by factors 4 and 5 . However, the manual minutiae cannot avoid errors related to limited overlap and nonlinear distortions (factors 2 and 3). Therefore, we will analyze our algorithms' sensitivity to factors $3-5$ in this section. The nonlinear distortions (factor 2) can be included into minutiae errors (factor 5). To measure the influences of these three factors, we simulated them on the 1000 minutiae sets that we manually extracted from MCYT. We used the parameter setting in Section II-C and the direct matching algorithm to perform the simulations.

\section{A. Limited Overlap Between the Reference and Test Fingerprints}

During the verification phase, the finger may be placed at different locations and in different angles on the sensor, which results in a limited overlap between the reference and test fingerprints. In both the SML and SMO algorithms, all the extracted minutiae are used in the fingerprint representation; therefore, a limited overlap between a fingerprint pair will cause an insufficient percentage of matching minutiae, and will lead to a reduced performance.

In order to study this effect, we performed simulations where minutiae in a certain area were removed from the extracted minutia set. During the simulation, based on the minutiae location, we removed a fraction of $p$ minutiae at the top of a fingerprint to obtain the reference minutia set. For the test minutiae set, we removed a fraction of $p$ minutiae from the same fingerprint at the bottom (see Fig. 16). All the corresponding minutiae are without any errors on both location and orientation. After the minutiae removals, the corresponding minutiae fraction $P_{\text {corr }}$ between the reference and test fingerprints is

$$
P_{\text {corr }}=\frac{1-2 p}{1-p} \text {. }
$$

The matching results for different percentage $p$ are shown in Fig. 17.

From the results we can see that when the removal percentage is below 20\% (in which case the corresponding minutiae percentage $P_{\text {corr }}$ is above $75 \%$ ), it will hardly cause any error recognition in both the SML and SMO algorithms. When $p$ increases, the performances of both algorithms degrade. Compared with SMO, SML is more robust to the limited overlap between a fingerprint pair.

\section{B. Missing and Spurious Minutiae}

An unreliable minutiae extractor or bad quality fingerprints can bring a great number of missing and spurious minutiae, 

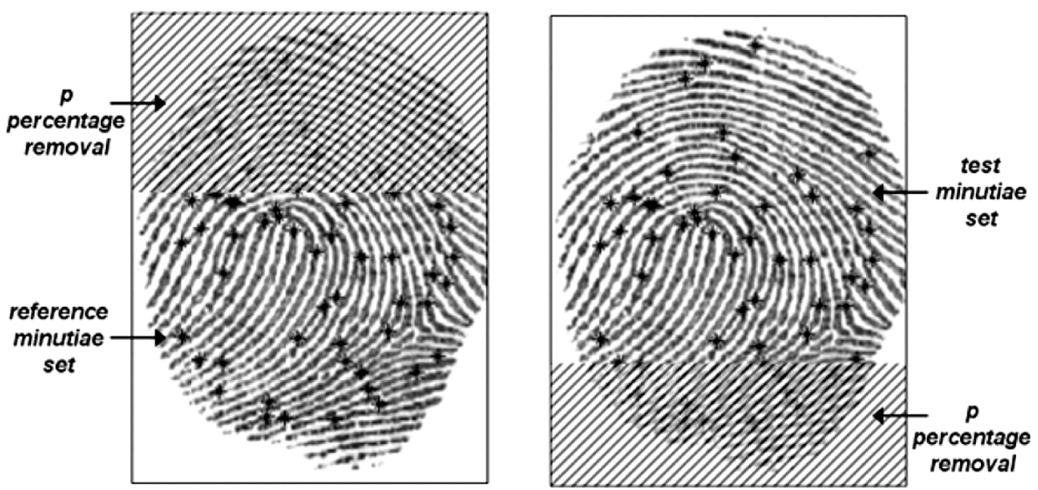

Fig. 16. Creation of limited overlap between a fingerprint pair.

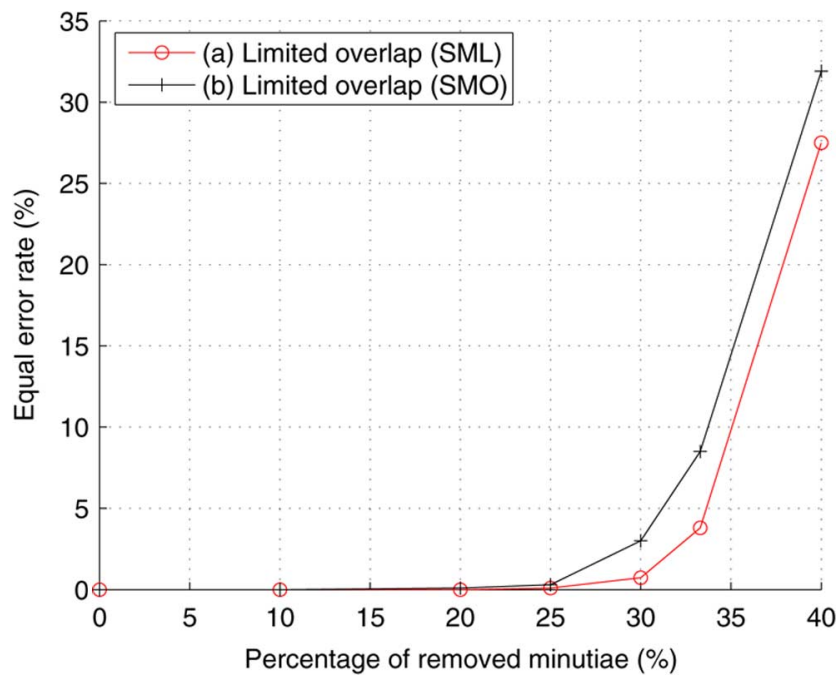

Fig. 17. Simulation of limited overlap between the reference and test fingerprints.

which will lead to an insufficient number of corresponding minutiae, and then lead to a reduced performance. During the simulation, we first discarded a fraction of $p$ randomly chosen minutiae from a minutiae set to obtain a reference minutiae set. Next, we discarded another fraction of $p$ minutiae from the original minutiae set to obtain the test minutiae set. The matching results for different $p$ are shown in Fig. 18.

Similar as the simulation results from the limited overlap case, missing and spurious minutiae do not influence the EERs if the removed fraction is below 20\%. When $p$ increases, the performances of both algorithms degrade. When $p$ is relatively small (below to 35\%), SML performances better than SMO. When $p$ is bigger, SML degrades faster than SMO, which leads to a worse performance.

\section{Errors on Minutiae Location and Orientation}

Many factors can cause errors on minutiae location and orientation, such as an unreliable minutiae extractor, noisy fingerprint images, and elastic deformations of fingerprints. The latter one is even unavoidable for manually extracted minutiae. During the simulation, the original minutiae sets are used as reference minutiae sets. Then we add zero-mean Gaussian distributed noise to the minutiae location or/and orientation to build test minutiae sets.

For the SML algorithm, we simulated the case of errors on the minutiae location. For the SMO algorithm, we simulated three

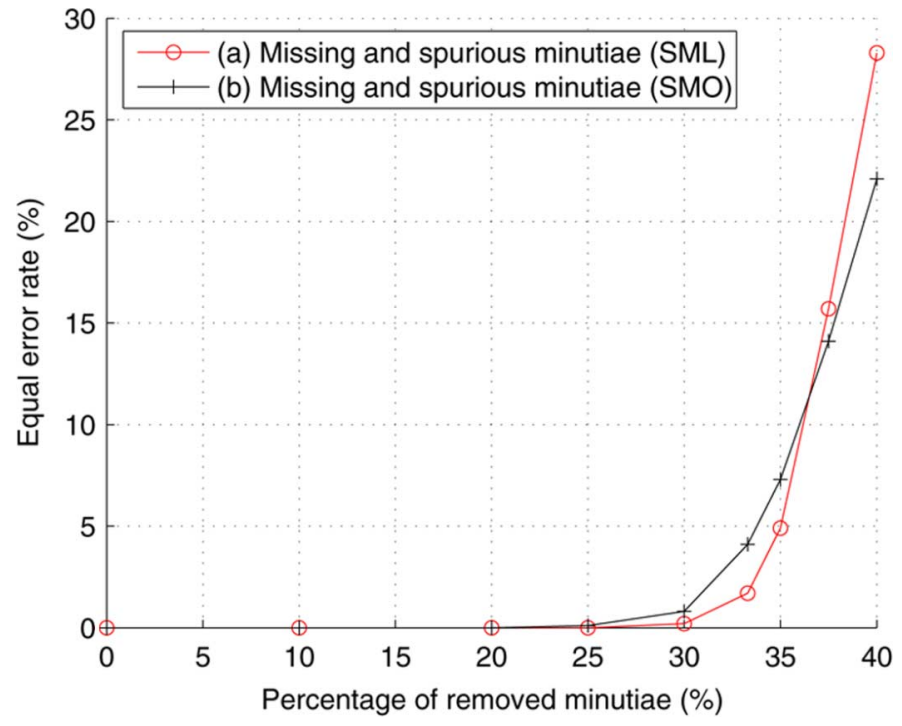

Fig. 18. Simulation of missing and spurious minutiae.

cases: (a) errors only on the minutiae location; (b) errors only on the minutiae orientation; and (c) errors on both the minutiae location and orientation.

Fig. 19 shows the influence of minutiae location errors to SML and SMO (the errors that we added are measured by pixels, for our fingerprints, 1 pixel is about $0.05-\mathrm{mm}$ distance in reality). We can see that if the location errors are relatively small (the standard deviation $\sigma$ is smaller than 3 pixels), this will not cause any false recognition. When the errors increase, the performances of both SML and SMO degrade. When $\sigma$ is smaller than 7, SML performs better than SMO. When $\sigma$ is bigger than 7, SML degrades faster than SMO, and finally leads to a worse performance.

Fig. 20 shows the influence of different error cases to SMO. To simulate a similar error scale on minutiae location and orientation, we scaled to orientation such that 1 pixel error on the location corresponds to $0.04 \mathrm{rad}$ error on the orientation. Compared with cases (a) and (b), we can see that the errors on minutiae orientation cause much less performance degradation than the errors on minutiae location. The case (a) and (c) show that adding errors on orientation does not degrade the result greatly.

From Figs. 19 and 20, we also notice that when the errors become bigger (for example, $\sigma$ changes from 6 to 7), the EERs can degrade with $6 \%$. That is a big influence which cannot be ignored. 


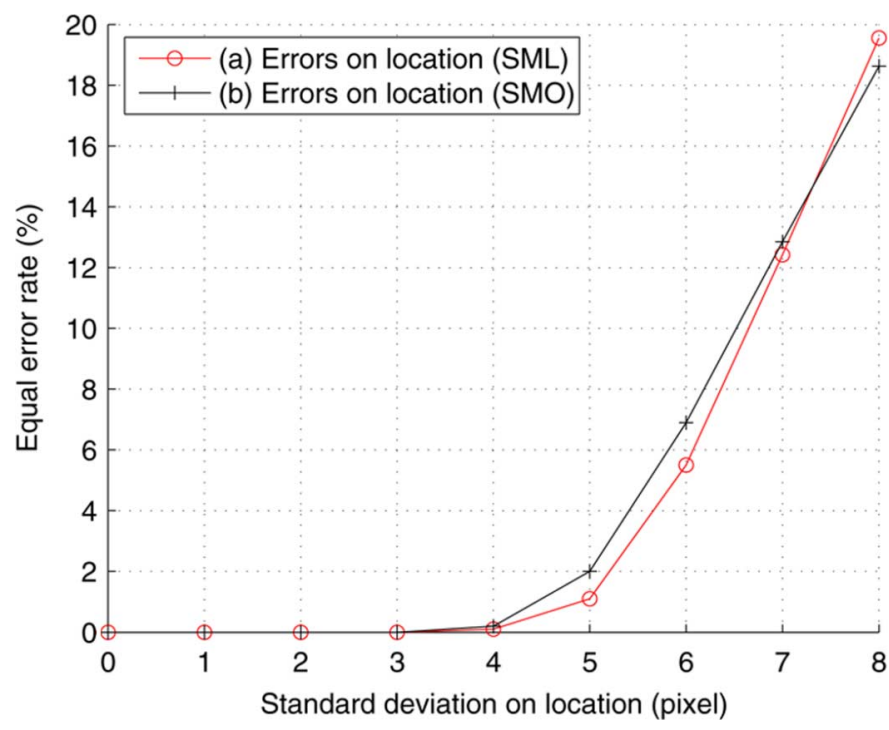

Fig. 19. Simulation of errors on the minutiae location (SML and SMO).

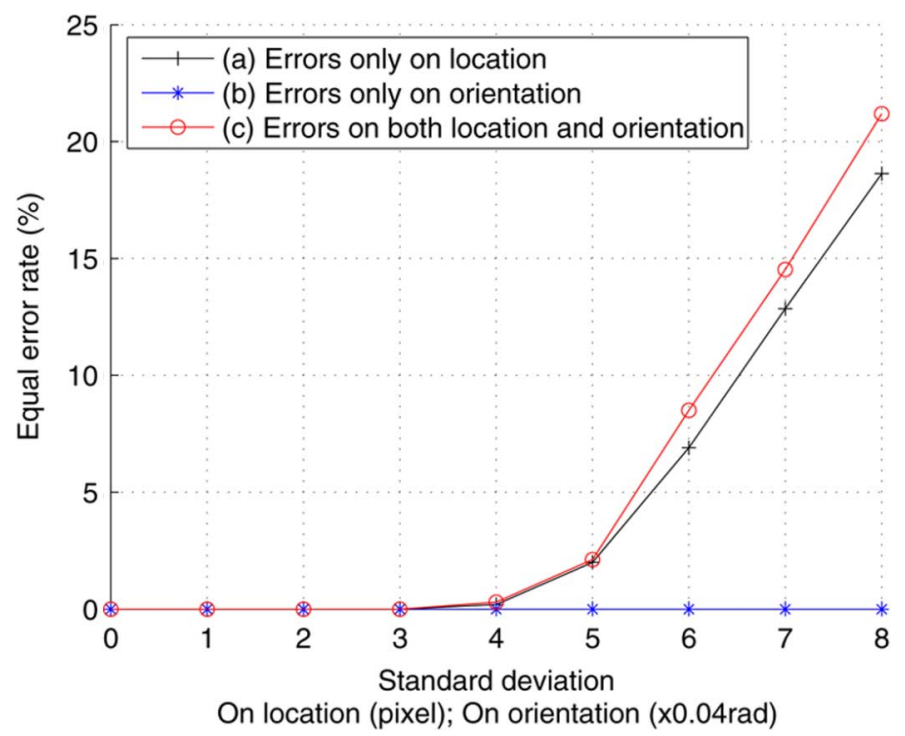

Fig. 20. Simulation of errors on the minutiae location and orientation (SMO).

\section{CONCLUSION}

The spectral minutiae representation is a novel method to represent a minutiae set as a fixed-length feature vector, which enables the combination of fingerprint recognition systems and template protection schemes. In order to be able to apply the spectral minutiae representation with a template protection scheme, for example based on a fuzzy extractor [27], the next step would be to extract bits that are stable for the genuine user and completely random for an arbitrary user. For example, we can apply 2-D Gabor filters for bit extraction, which has been used in iris codes [28]. Another possibility is to first apply additional dimensionality reduction by a combination of PCA and LDA and then apply single bit extraction according to the reliable component scheme or multibit extraction [29].

The spectral minutiae representation avoids the difficulties of minutiae registration by representing a minutiae set as a translation-invariant spectrum, in which rotation and scaling become translations, which can be easily compensated for. Moreover, this method is compatible with the large number of existing minutiae databases and the additional cost to integrate this new scheme is relatively low.

This paper introduces two spectral minutiae representation methods: the SML and the SMO. Both algorithms are evaluated using a correlation-based spectral minutiae matching algorithm (direct matching) and a Fourier-Mellin matching algorithm. From the experiments, the direct matching algorithm gives a better performance. Comparing SML and SMO, SML shows better results if the fingerprints are of good quality and the minutiae extractor is reliable, while SMO is more robust against the minutiae noise. After investigating the relation between the SML and SMO results, a matching score based fusion is applied, which obtains better results. Finally, a method using SPs showing a better performance is presented.

In this paper, we also discussed three factors that can degrade our algorithms' performances: limited overlap between the reference and test fingerprints; missing and spurious minutiae; errors on minutiae location and orientation. It shows that in general SML is more robust to all three factors. However, if the percentage of missing and spurious minutiae is too high $(p>35 \%)$, or the errors on minutiae location are too big [standard deviation $\sigma>7$ (pixel)], then SMO shows a better performance. Therefore, in case the fingerprints have good quality and/or the minutiae extractor is reliable, SML shows a better recognition performance. Overall, the performance can be improved by implementing fusion of SML and SMO. However, these three factors are unavoidable in a fingerprint recognition system. To cope with the limited overlaps and to be more robust against the minutiae errors are topics of further research.

\section{REFERENCES}

[1] D. Maltoni, D. Maio, A. Jain, and S. Prabhakar, Handbook of Fingerprint Recognition.. New York: Springer, 2003.

[2] A. Bazen, R. Veldhuis, and S. Gerez, "Hybrid fingerprint matching using minutiae and shape," in Computer Aided Intelligent Recognition Techniques and Applications. . Hoboken, NJ: Wiley, 2004.

[3] A. Jain, L. Hong, and R. Bolle, "On-line fingerprint verification," IEEE Trans. Pattern Anal. Mach. Intell., vol. 19, no. 4, pp. 302-314, Apr. 1997.

[4] A. Bazen and S. Gerez, "Fingerprint matching by thin-plate spline modelling of elastic deformations," Pattern Recognit., vol. 36, no. 8, pp. 1859-1867, Aug. 2003.

[5] United States Visitor and Immigrant Status Indicator Technology Program (US-VISIT) [Online]. Available: http://www.dhs.gov/us-visit

[6] P. Tuyls, A. Akkermans, T. Kevenaar, G. Schrijen, A. Bazen, and R. Veldhuis, "Practical biometric authentication with template protection," in AVBPA, 2005, pp. 436-446.

[7] A. Juels, , P. Tuyls, B. Skoric, and T. Kevenaar, Eds., "Fuzzy commitment," in Security With Noisy Data-On Private Biometrics, Secure Key Storage and Anti-Counterfeiting. New York: Springer, 2007, ch. 3, pp. $45-56$.

[8] K. Nandakumar, A. Jain, and S. Pankanti, "Fingerprint-based fuzzy vault: Implementation and performance," IEEE Trans. Inf. Forensics Security, vol. 2, no. 4, pp. 744-757, Dec. 2007.

[9] D. Casasant and D. Psaltis, "Position, rotation, and scale invariant optical correlation," Appl. Opt., vol. 15, no. 7, pp. 1795-1799, Jul. 1976.

[10] S. Derrode and F. Ghorbel, "Robust and efficient Fourier-Mellin transform approximations for gray-level image reconstruction and complete invariant description," Computer Vision and Image Understanding: CVIU, vol. 83, no. 1, pp. 57-78, Jul. 2001.

[11] Q. Chen, M. Defrise, and F. Deconinck, "Symmetric phase-only matched filtering of fourier-mellin transform for image registration and recognition," IEEE Trans. Pattern Anal. Mach. Intell., vol. 16, no. 12, pp. 1156-1168, Dec. 1994.

[12] Y. Sheng and H. Arsenault, "Experiments on pattern recognition using invariant Fourier-Mellin descriptors," J. Opt. Soc. Amer. A, vol. 3, no. 6, pp. 771-776, Jun. 1986.

[13] F. Ghorbel, "A complete invariant description for gray-level images by the harmonic analysis approach," Pattern Recognit. Lett., vol. 15, no. 10, pp. 1043-1051, 1994. 
[14] R. Bolle, J. H. Connell, S. Pankanti, N. K. Ratha, and A. W. Senior, Guide to Biometrics.. New York: Springer Verlag, 2003.

[15] ISO/IEC 19794-2, Information Technology-Biometric Data Interchange Format-Part 2: Finger Minutiae Data 2005.

[16] R. Bracewell, The Fourier Transform and Its Applications, 3rd ed. New York: McGraw-Hill, 1999

[17] Y. Sheng and J. Duvernoy, "Circular-fourier-radial-mellin transform descriptors for pattern recognition," J. Opt. Soc. Amer. A, vol. 3, no. 6, pp. 885-888, Jun. 1986.

[18] J. Ortega-Garcła et al., "MCYT baseline corpus: A bimodal biometric database," IEE Proc. Vision, Image and Signal Processing, vol. 150, no. 6, pp. 395-401, 2003.

[19] D. Maio, D. Maltoni, R. Cappelli, J. Wayman, and A. Jain, "FVC2000: Fingerprint verification competition," IEEE Trans. Pattern Anal. Mach. Intell., vol. 24, no. 3, pp. 402-412, Mar. 2002.

[20] D. Maio, D. Maltoni, R. Cappelli, J. Wayman, and A. Jain, FVC2002: Second Fingerprint Verification Competition, vol. 3, pp. 811-814, Aug. 2002.

[21] VeriFinger SDK [Online]. Available: http://www.neurotechnologija.com/

[22] A. Bazen and S. Gerez, "Segmentation of fingerprint images," in Proc. 12th Annual Workshop on Circuits, Systems and Signal Processing (ProRISC 2001), Veldhoven, The Netherlands, Nov. 2001.

[23] A. A. Ross, K. Nandakumar, and A. K. Jain, Handbook of Multibiometrics (International Series on Biometrics).. Secaucus, NJ: SpringerVerlag New York, Inc., 2006.

[24] A. Ross, A. K. Jain, and J.-Z. Qian, "Information fusion in biometrics," in AVBPA'01: Proc. 3rd Int. Conf. Audio- and Video-Based Biometric Person Authentication, London, U.K., 2001, pp. 354-359.

[25] E. Henry, Classification and Uses of Finger Prints.. London: Routledge, 1900.

[26] A. Jain, S. Prabhakar, L. Hong, and S. Pankanti, "Filterbank-based fingerprint matching," IEEE Trans. Image Process., vol. 9, no. 5, pp. 846-859, May 2000.

[27] Y. Dodis, L. Reyzin, and A. Smith, , P. Tuyls, B. Skoric, and T. Kevenaar, Eds., "Fuzzy extractors," in Security With Noisy Data-On Private Biometrics, Secure Key Storage and Anti-Counterfeiting. New York: Springer, 2007, ch. 3, pp. 45-56.

[28] J. Daugman, "How iris recognition works," IEEE Trans. Circuits Syst. Video Technol., vol. 14, no. 1, pp. 21-30, Jan. 2004.

[29] C. Chen, R. Veldhuis, A. Akkermans, and T. Kevenaar, "Biometric binary string generation with detection rate optimized bit allocation," in Proc. IEEE Computer Society Conf. Computer Vision and Pattern Recognition-Workshop on Biometrics, Anchorage, AK, 2008.

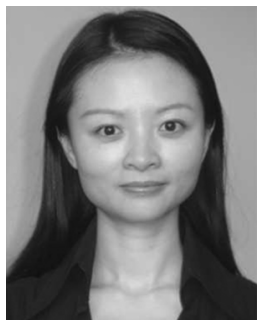

Haiyun Xu received the B.E. and M.E. degrees in electrical engineering from Beijing University of Technology, China, in 2000 and 2003, respectively. In 2005, she began to pursue the Ph.D. degree with the Department of Electrical Engineering, University of Twente, The Netherlands.

In 2003, she worked with Sony in Beijing to work on the Chinese input method for Sony Play Station 2. In 2004, she joined Siemens Ltd. China to work on mobile phone software development for one year. Her current research focuses on the integration of biometric identification in security systems. Her research interests include biometrics, pattern recognition, signal processing, and security.

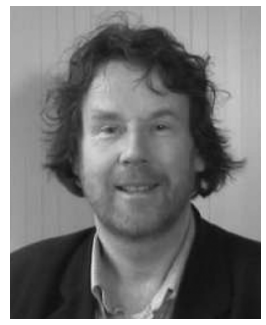

Raymond N. J. Veldhuis received the engineering degree in electrical engineering from the University of Twente, The Netherlands, in 1981. In 1988, he received the Ph.D. degree from Nijmegen University.

From 1982 to 1992, he worked as a researcher at Philips Research Laboratories in Eindhoven, The Netherlands, in various areas of digital signal processing, such as audio and video signal restoration and audio source coding. From 1992 to 2001, he worked at the Institute of Perception Research (IPO) Eindhoven in speech signal processing and speech synthesis. He is now an Associate Professor at the University of Twente, working in the fields of biometrics, pattern recognition, and signal processing.

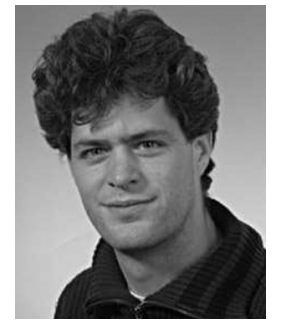

Asker M. Bazen received the Ph.D. degree in electrical engineering from the University of Twente, The Netherlands, in 2002, for his research on fingerprint recognition.

He supervised several biometric research projects at the University of Twente. These projects include research on fingerprint recognition, face recognition, and grip-pattern recognition for securing a smart gun against unauthorized use. Currently he is CEO and owner of Uniqkey Biometrics, a company that is specialized in fingerprint recognition technology for very fast and reliable search through large fingerprint databases.

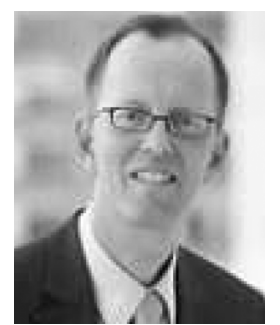

Tom A. M. Kevenaar graduated in 1986 from the Technical University of Eindhoven, The Netherlands. After joining Philips to work on switched-mode power supplies, he returned to Eindhoven University in 1989, and in 1993 obtained the Ph.D. degree in the field of computer-aided design for analogue circuits.

After one more year in Eindhoven to work on a method to determine the periodic steady-state solution of nonlinear systems and to develop formal methods for automated selection of optimal test measurements for analogue circuits, he joined Hitachi Central Research Laboratory, Tokyo, Japan, in early 1994, where he developed numerical optimization methods to be used in designing analogue circuits. In 1996, he joined Philips Research where he guided the development of a large variety of analogue design tools such as analogue/radio-frequency circuit simulators, optimization tools, fault simulation tools, etc. In 2001, he moved to the field of cryptography with a focus on low-power cryptographic algorithms and standards, and slightly later became involved in privacy enhancing technologies for biometric applications. In 2008, he cofounded a start-up company called priv-ID, which develops and sells products to protect the privacy of biometric information.

Dr. Kevenaar has over 40 publications in international conferences and journals and is co-editor of the book Security with Noisy Data (Springer, 2007).

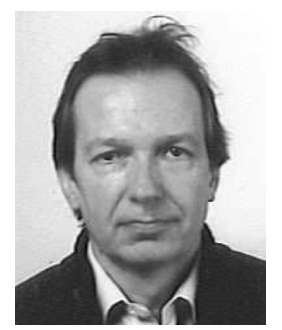

Ton A. H. M. Akkermans was born in 1959, in Breda, the Netherlands. He received the M.Sc. degree in theoretical electrical engineering (Digital Signal Processing Department) from the Eindhoven University of Technology.

In 1982, he joined Philips Research Laboratories, where he worked on geometrical and diffraction optics, optical alignment systems, projection TV, wafer steppers, and computer-generated holographics. Since 1987, he has been engaged at Philips Optical Storage as a Senior System Architect and was responsible for the mechatronical architectures of CD-R/RW and DVD systems until mid 2000. In 2000, he joined the PACMan group (Processing Architectures for Content Management) within Philips Research and developed architectures for copy control, key management systems for digital rights management (DRM), and watermarking techniques. Currently he is working as a Principal Scientist on biometric systems and privacy protection architectures related to biometric identification. He has published a number of papers and reports and is the holder of several patents in the above-mentioned fields.

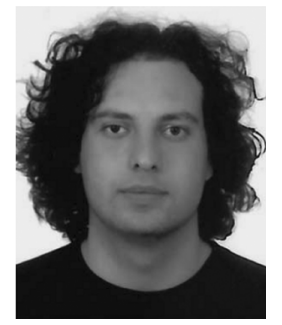

Berk Gokberk received the B.S., M.S., and Ph.D degrees in computer science from Bogazici University, Istanbul, Turkey, in 1999, 2001, and 2006, respectively.

From 2006 to 2008, he worked as a Senior Scientist at Philips Research Laboratories-Information System Security Division, Eindhoven, The Netherlands. He is currently with the Signals and Systems Group at the Department of Electrical Engineering Mathematics and Computer Science, University of Twente, The Netherlands. His research interests include biometrics, 2-D/3-D face recognition, pattern recognition, and computer vision. 